\title{
Detection and Oncologic Outcomes of Head and Neck Squamous Cell Carcinoma of Unknown Primary Origin
}

\author{
MAXWELL Y. LEE ${ }^{1}$, NICOLE FOWLER ${ }^{2}$, DAVID ADELSTEIN ${ }^{3}$, \\ SHLOMO KOYFMAN $^{4}$, BRANDON PRENDES ${ }^{5}$ and BRIAN B. BURKEY ${ }^{5}$ \\ ${ }^{1}$ Cleveland Clinic Lerner College of Medicine of Case Western Reserve University, \\ EC-10 Cleveland Clinic, Cleveland, OH, U.S.A.; \\ ${ }^{2}$ Department of Otolaryngology-Head and Neck Surgery, ENT Institute, \\ University Hospitals Cleveland Medical Center, South Euclid, OH, U.S.A.; \\ ${ }^{3}$ Department of Hematology and Medical Oncology, Taussig Cancer Institute, \\ Cleveland Clinic, Cleveland, OH, U.S.A.; \\ ${ }^{4}$ Department of Radiation Oncology, Taussig Cancer Institute, Cleveland Clinic, Cleveland, OH, U.S.A.; \\ ${ }^{5}$ Head and Neck Institute, Cleveland Clinic, Cleveland, OH, U.S.A.
}

\begin{abstract}
Background/Aim: To assess factors that predict detection of tumors and oncologic outcomes in head and neck squamous cell carcinoma of unknown primary (SCCUP). Patients and Methods: This was a retrospective cohort study at a single tertiary care institution. Results: The primary site was detected at examination under anesthesia (EUA) in $92(51.1 \%)$ patients. The primary site was detected by directed biopsies in 60 (65\%), palatine tonsillectomy in $28(30.4 \%)$, and lingual tonsillectomy in 4 patients $(4.3 \%)$. Four of eight lingual tonsillectomies were positive (50\%). Primary locations included: palatine tonsils (51, 28.3\%), base of tongue (37, 20.6\%), larynx (4, 2.2\%), oral cavity (3, $1.67 \%)$ and nasopharynx $(1,0.6 \%)$. Human papillomavirus $(H P V)$ positive status $(H R=0.26, p=0.004)$ and treatment with chemoradiation (CRT) $(H R=0.38, p=0.004)$ were associated with better disease free survival (DFS). Conclusion: A primary site was located after aggressive investigation in approximately half of the patients. More research is warranted towards the use of lingual tonsillectomy. Predictors of favorable prognosis included $H P V$ positive status and treatment with CRT.
\end{abstract}

Head and neck squamous cell carcinoma of unknown

This article is freely accessible online.

Correspondence to: Brian B. Burkey, Cleveland Clinic Head and Neck Institute. 9500 Euclid Ave A71, Cleveland, OH 44106, U.S.A. Tel: +1 2163125645, e-mail: burkeyb1@ccf.org

Key Words: Head and neck cancer, unknown primary, human papillomavirus, oropharyngeal cancer, survival. primary origin (SCCUP) accounts for 2-4\% of head and neck cancers $(1,2)$. These patients represent a challenging population to diagnose and treat. Currently, there is a lack of consensus about the optimal diagnosis and treatment strategy that creates a decreased ability to counsel and formulate care plans for these patients. The approach to diagnosis and treatment is variable and in many patients, a primary site is never identified (3).

Diagnostic evaluation of SCCUP following history, physical examination, and histological confirmation of squamous cell carcinoma (and HPV/p16 status) after lymph node fine needle aspiration (FNA) or biopsy is largely focused on determining the primary site. In-office flexible laryngoscopy is used to identify easily visualized lesions. Next, imaging using fluorodeoxyglucose positron emission tomography/computed tomography (FDG-PET/CT) can help to locate the primary site in a minority of patients $(4,5)$. More recently, transoral robotic surgery lingual tonsillectomy has emerged as another tool to diagnose SCCUP that may increase the primary detection rate by approximately $20 \%(6,7)$. When testing is unable to detect a primary site, a final diagnosis of unknown primary is made. Several theories attempt to explain the existence of SCCUP: the primary site may undergo regression or destruction (e.g. immune mediated), the primary site may be undetectable due to location (e.g. hidden in the crypt epithelium of the palatine or lingual tonsils), or the primary site may be undetectable due to small size (8).

Treatment for SCCUP is focused on controlling disease in the neck as well as preventing the emergence of a primary cancer. Fortunately, modern techniques including neck dissection followed by radiation with or without chemotherapy and primary chemoradiation result in excellent oncological outcomes (9). 
The landscape and understanding of SCCUP is quickly evolving. In the recent years, an increasing portion of SCCUP has been attributed to human papilloma virus (HPV) in concordance with a similar increase in HPV associated oropharyngeal squamous cell carcinoma (SCC) and its propensity to present with a small primary tumor and advanced nodal involvement (10-12). Furthermore, advanced imaging and biopsy techniques have become available, decreasing the number of patients with true SCCUP $(4,7)$. Lastly, the relative rarity of SCCUP has prevented high quality prospective studies, causing variability in detection and treatment standards among different institutions. In this study, we present our single institution's experience with SCCUP over a period of 18 years with a focus on methods of primary site detection and predictors of positive prognosis in these patients.

\section{Patients and Methods}

Patient selection and data collection. We conducted an institutional review board (IRB) approved, retrospective review of patients at a single tertiary care institution between January 1, 2000 and December 31, 2018. All patients with a possible diagnosis of SCCUP were screened. This included all patients with a diagnosis of unknown primary head and neck cancer from an institutional tumor registry along with all patients entered into an institutionwide head and neck cancer database with a diagnosis of unknown primary, T0, TX, or T1/N+ nasopharynx, oral cavity, oropharynx, hypopharynx, or laryngeal cancer. All patients had to have a confirmed histological diagnosis of squamous cell carcinoma.

Patients where an obvious primary site was not located after an in-office exam by a head and neck surgeon were included in the final study. An in-office exam consisted of a comprehensive physical exam and flexible laryngoscopy or mirror exam. Patients with a previous diagnosis of squamous cell carcinoma, who were unable to undergo in office examination, or received care outside of the Cleveland Clinic were excluded from the study.

All data was collected from the medical records of these patients and stored in an online, institutional review board approved, REDCap database. This included: demographic characteristics (age, ethnicity, sex), smoking and alcohol status, pre-existing functional status (voice, dysphagia, feeding tube), diagnostic workup (procedures performed, location of biopsy, location of tumor), tumor characteristics (location, stage, cell differentiation, HPV status), treatment characteristics (treatment modality, date, complications), recurrence, and follow-up. The AJCC 7th edition staging was used throughout this study.

Statistical analysis. All analyses were performed using the $\mathrm{R}$ statistical software (version 3.6.1, R Foundation for Statistical Computing). Descriptive statistics were used to describe demographic and treatment data. Quantitative variables were reported as means with $95 \%$ confidence intervals (CI) or medians with interquartile ranges, unless otherwise noted. Differences between groups were assessed with Student's $t$-test or Kruskal Wallis rank sum test. Categorical data was represented as counts with percentages. Differences between groups were assessed with Chi squared test. Survival was modelled with Kaplan Meier analysis and differences were assessed using the log-rank test. Overall survival (OS) was defined as time from treatment to death whereas disease free survival (DFS) was defined as time from treatment to recurrence or death. Risk factors for our endpoint of DFS were assessed using multi-variable Cox proportional hazards regression models and presented as calculated hazard ratios (HR) and 95\% CIs. Predictive factors for primary detection were assessed with multivariable logistic regression and presented as odds ratios (OR) and $95 \%$ CIs.

\section{Results}

A total of 489 patients were screened for inclusion, 180 of whom met the criteria for the study and were designated as SCCUP based on lack of an obvious primary site after inoffice examination by a head and neck surgeon. The median follow-up time was 39.1 months (range=1.6-172.9). The average patient age was $60(\mathrm{SD}=8.6)$ years and $156(86.7 \%)$ patients were male. The majority of patients were either current or former smokers $(144,63.3 \%)$ and $38(21.1 \%)$ patients were current or former heavy alcohol users. Disease was clinically staged as $\mathrm{N} 1$ (cN1) in 30 patients $(16.7 \%)$, cN2 in 127 patients $(70.6 \%), \mathrm{cN} 3$ in 23 patients $(12.8 \%)$ (Table I).

At the time of histological diagnosis, human papilloma virus (HPV) status was inferred in most patients by testing for either p16 immunohistochemical stain or HPV DNA with fluorescence in situ hybridization testing (FISH). In total, $\mathrm{HPV} / \mathrm{p} 16$ was positive in $150(83.3 \%)$, negative in 14 $(7.8 \%)$, and untested in $16(8.9 \%)$ patients. Of the positive patients, 92 were identified by p16 staining $(61.3 \%), 17$ by fluorescence in situ hybridization assay (11.3\%), and 41 by both methods (27.3\%). Epstein Barr virus (EBV) testing was not routinely performed in our cohort.

To detect the primary site, $133(73.9 \%)$ patients received PET prior to direct laryngoscopy (DL) with directed biopsies. PET identified an area of abnormal uptake causing suspicion for a primary site in $64(48 \%)$ patients, but a primary site was only found in $40(62.5 \%)$ of these patients. Next, all patients received operative DL with biopsies. At the time of surgery, eight $(4.4 \%)$ patients received unilateral lingual tonsillectomy and $87(48.3 \%)$ received palatine tonsillectomy (36 bilateral, 51 unilateral); 43 (23.9\%) patients had a history of previous palatine tonsillectomy. Pathologically tested sites in order of frequency were: base of tongue $(141,78.3 \%)$, palatine tonsil $(107,59.4 \%)$, pharyngeal walls $(46,25.6 \%)$, and larynx $(20,11.1 \%)$.

The location of the primary site was detected at surgery in $92(51.1 \%)$ patients. Primary locations in order of frequency included: palatine tonsils $(51,28.3 \%)$, base of tongue $(37,20.6 \%)$, larynx $(4,2.2 \%)$, oral cavity $(3,1.67 \%)$ and nasopharynx $(1,0.6 \%)$. The primary site was unilateral to the presenting metastatic lymph node in 87 patients $(89 \%)$, contralateral in two patients $(2 \%)$, and bilateral in 6 patients $(6 \%)$. Additionally, three patients (3\%) initially presented with bilateral nodal disease. Primary site was 
Table I. Patient and treatment characteristics stratified by HPV status.

\begin{tabular}{|c|c|c|c|c|c|c|c|c|c|}
\hline & \multicolumn{2}{|c|}{$\begin{array}{c}\text { Total } \\
(\mathrm{N}=180)\end{array}$} & \multicolumn{2}{|c|}{$\begin{array}{l}\text { HPV negative } \\
\quad(\mathrm{n}=14)\end{array}$} & \multicolumn{2}{|c|}{$\begin{array}{l}\text { HPV not tested } \\
\quad(n=16)\end{array}$} & \multicolumn{2}{|c|}{$\begin{array}{l}\text { HPV positive } \\
\quad(\mathrm{n}=150)\end{array}$} & \multirow[t]{2}{*}{$p$-Value } \\
\hline & $\mathrm{N}$ & $\%$ & $\mathrm{n}$ & $\%$ & $\mathrm{n}$ & $\%$ & $\mathrm{n}$ & $\%$ & \\
\hline Ethnicity: Caucasian & 168 & $93.33 \%$ & 12 & $85.71 \%$ & 15 & $93.75 \%$ & 141 & $94.00 \%$ & 0.345 \\
\hline Age (mean, SD) & 59.66 & 8.56 & 61.71 & 5.01 & 59.66 & 9.59 & 59.48 & 8.73 & 0.65 \\
\hline Gender: Male & 156 & $86.67 \%$ & 11 & $78.57 \%$ & 15 & $93.75 \%$ & 130 & $86.67 \%$ & 0.475 \\
\hline Current or former smoker & 114 & $63.33 \%$ & 11 & $78.57 \%$ & 13 & $81.25 \%$ & 90 & $60.00 \%$ & 0.415 \\
\hline Current or former heavy alcohol user & 38 & $21.11 \%$ & 6 & $42.86 \%$ & 6 & $37.50 \%$ & 26 & $17.33 \%$ & 0.02 \\
\hline Clinical $\mathrm{N}$ stage & & & & & & & & & 0.04 \\
\hline 1 & 30 & $16.67 \%$ & 2 & $14.29 \%$ & 3 & $18.75 \%$ & 25 & $16.67 \%$ & \\
\hline 2 & 127 & $70.56 \%$ & 6 & $42.86 \%$ & 10 & $62.50 \%$ & 111 & $74.00 \%$ & \\
\hline 3 & 23 & $12.78 \%$ & 6 & $42.86 \%$ & 3 & $18.75 \%$ & 14 & $9.33 \%$ & \\
\hline Unilateral lingual tonsillectomy & 10 & $5.56 \%$ & 0 & $0.00 \%$ & 0 & $0.00 \%$ & 10 & $6.67 \%$ & 0.347 \\
\hline Palatine tonsillectomy & & & & & & & & & 0.135 \\
\hline None & 50 & $27.78 \%$ & 4 & $28.57 \%$ & 5 & $31.25 \%$ & 41 & $27.33 \%$ & \\
\hline Previous & 43 & $23.89 \%$ & 2 & $14.29 \%$ & 1 & $6.25 \%$ & 40 & $26.67 \%$ & \\
\hline Unilateral & 51 & $28.33 \%$ & 2 & $14.29 \%$ & 5 & $31.25 \%$ & 44 & $29.33 \%$ & \\
\hline Bilateral & 36 & $20.00 \%$ & 6 & $42.86 \%$ & 5 & $31.25 \%$ & 25 & $16.67 \%$ & \\
\hline PET & 133 & $73.89 \%$ & 12 & $85.71 \%$ & 11 & $68.75 \%$ & 110 & $73.33 \%$ & \\
\hline Location of primary & & & & & & & & & $<0.01$ \\
\hline Base of tongue & 37 & $20.56 \%$ & 1 & $7.14 \%$ & 0 & $0.00 \%$ & 36 & $24.00 \%$ & \\
\hline Tonsil & 51 & $28.33 \%$ & 0 & $0.00 \%$ & 0 & $0.00 \%$ & 51 & $34.00 \%$ & \\
\hline Larynx & 4 & $2.22 \%$ & 0 & $0.00 \%$ & 4 & $25.00 \%$ & 0 & $0.00 \%$ & \\
\hline Oral cavity & 3 & $1.67 \%$ & 1 & $7.14 \%$ & 1 & $6.25 \%$ & 1 & $0.67 \%$ & \\
\hline Nasopharynx & 1 & $0.56 \%$ & 0 & $0.00 \%$ & 0 & $0.00 \%$ & 1 & $0.67 \%$ & \\
\hline Not detected & 81 & $45.00 \%$ & 9 & $64.29 \%$ & 11 & $68.75 \%$ & 61 & $40.67 \%$ & \\
\hline Treatment & & & & & & & & & $<0.01$ \\
\hline CRT & 100 & $55.56 \%$ & 6 & $42.86 \%$ & 4 & $25.00 \%$ & 90 & $60.00 \%$ & \\
\hline XRT & 20 & $11.11 \%$ & 1 & $7.14 \%$ & 2 & $12.50 \%$ & 17 & $11.33 \%$ & \\
\hline $\mathrm{CRT}+\mathrm{LND}$ & 36 & $20.00 \%$ & 6 & $42.86 \%$ & 3 & $18.75 \%$ & 27 & $18.00 \%$ & \\
\hline XRT+LND & 17 & $9.44 \%$ & 0 & $0.00 \%$ & 4 & $25.00 \%$ & 13 & $8.67 \%$ & \\
\hline LND & 7 & $3.89 \%$ & 1 & $7.14 \%$ & 3 & $18.75 \%$ & 3 & $2.00 \%$ & \\
\hline
\end{tabular}

HPV, Human papilloma virus; PET, positron emission tomography scan; CRT, chemoradiotherapy; XRT, radiotherapy; LND, lymph node dissection. Bold values indicate statistical significance.

detected by biopsies in 60 patients $(65 \%)$, palatine tonsillectomy in $28(30.4 \%)$, or lingual tonsillectomy in four $(4.3 \%)$. Of note, four of eight $(50 \%)$ diagnostic lingual tonsillectomies were positive for a primary site. Two lingual tonsillectomies were performed for treatment purposes. On multivariable analysis, controlling for HPV status, lack of suspicious findings on PET $(\mathrm{OR}=0.42, p=0.04)$ and previous palatine tonsillectomy $(\mathrm{OR}=0.3, p<0.01)$ were associated with inability to detect a primary site.

Treatment was concomitant chemoradiation (CRT) in 100 patients $(55.6 \%)$, radiation alone (XRT) in $20(11.1 \%)$, CRT with lymph node dissection (LND) in 36 (20\%), XRT with LND in $17(9.4 \%)$, or LND alone in seven patients $(3.9 \%)$. Chemotherapy was platinum based in 120 patients $(88 \%)$ or Cetuximab in 16 patients $(12 \%)$. The prescribed course of radiation for the majority of patients was $70 \mathrm{~Gy}$ given in 35 fractions. We found that overall survival (OS) was $91 \%$ at two years and $85 \%$ at five years. Similarly, disease free survival was $86 \%$ at two years and $77 \%$ at five years. On univariable analysis, we found that both OS and DFS were significantly higher among HPV positive as compared to HPV negative or untested patients (Figure 1; five-year DFS $84 \%$ vs. $32 \%$ and $56 \%$, respectively; $p<0.01)$. Expectedly, OS and DFS were also superior among patients with lower $\mathrm{N}$ stage disease (Figure 2; five-year DFS 82\% cN1, 81\% $\mathrm{cN} 2,54 \% \mathrm{cN} 3 ; p=0.04)$. With regards to treatment, CRT alone offered the highest OS and DFS compared to all other treatment modalities with five-year OS and DFS of $91.2 \%$ and $85.5 \%$, respectively (Figure 3 ). To determine the effect of our diagnostic workup on oncologic outcome, we examined whether the primary tumor was detected and found that OS and DFS were superior when a primary site was found (Figure $4 ; p<0.01$ ), although this was not significant upon multivariable analysis. Analysis of patients treated with platinum based chemotherapy versus Cetuximab showed no significant differences in OS or DFS. 

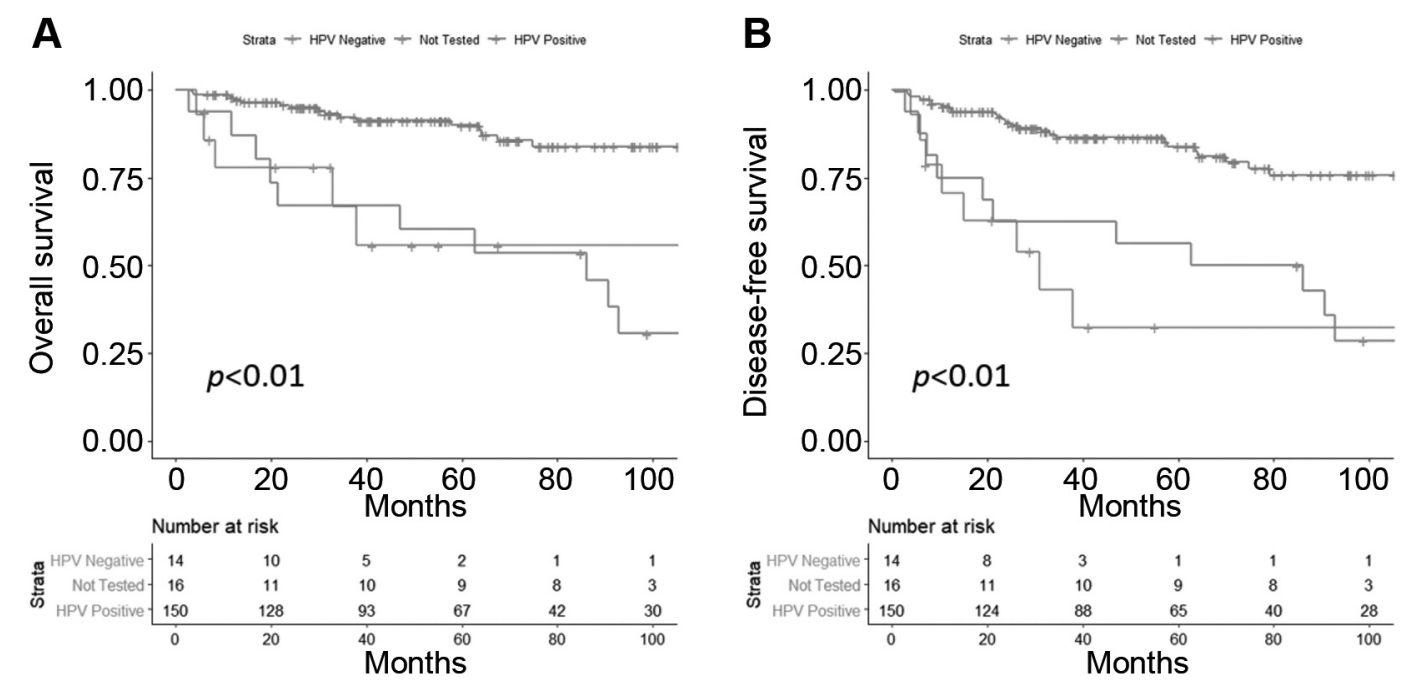

Figure 1. Overall (A) and disease free survival (B) among all patients stratified by HPV status. Vertical dashes represent censored patients.
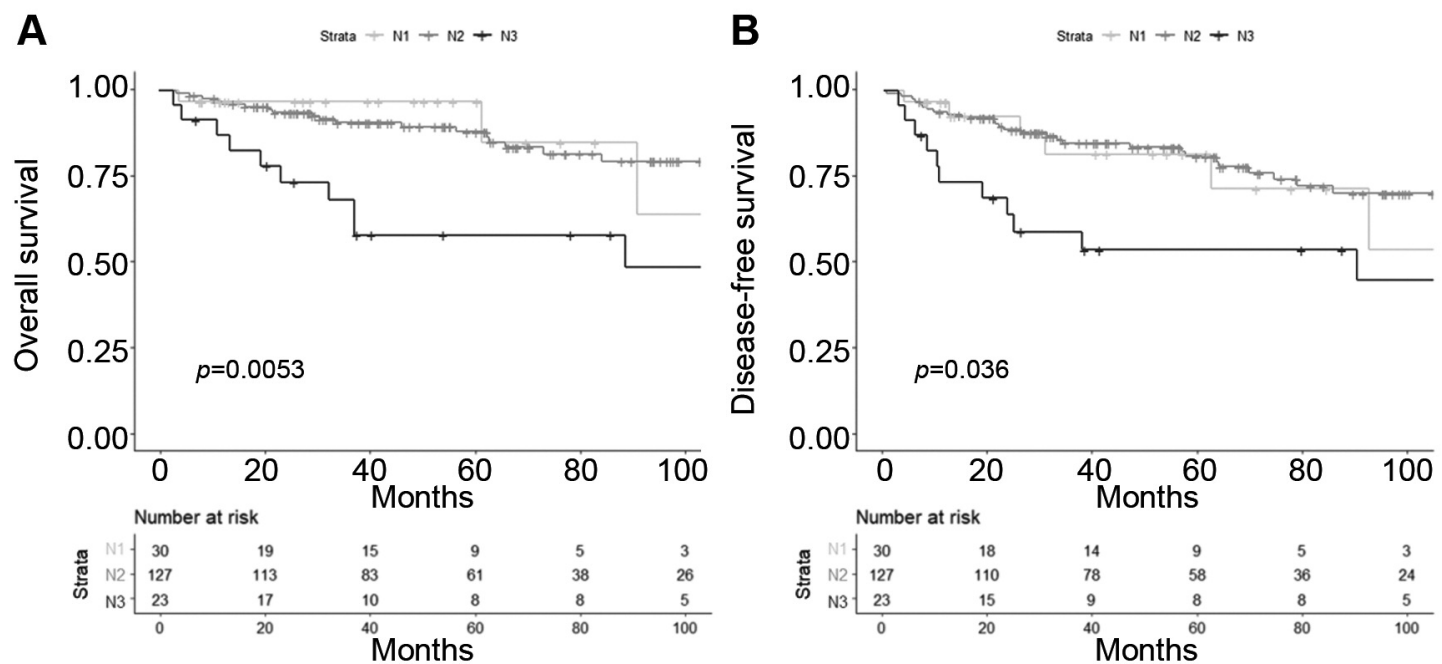

Figure 2. Overall (A) and disease free survival (B) among all patients stratified by clinical AJCC 7th edition $N$ stage. Vertical dashes represent censored patients.

Upon multivariable analysis of OS to account for $\mathrm{N}$ stage, HPV status, primary site detection, and treatment, $\mathrm{cN} 3$ disease was associated with increased risk of death (HR=7.06, $p=0.008)$. On multivariable analysis of DFS, positive HPV status (HR=0.26, $p=0.004)$ and treatment with CRT with or without LND (HR=0.38, $p=0.004$ ) were associated with decreased risk of failure (Table II).

Lastly, we performed a sensitivity analysis based upon our definition of SCCUP. While we defined SCCUP as patients without an obvious primary site after in-office examination by a head and neck surgeon, the population of patients without a located primary site prior to treatment can be considered as a more stringent definition of SCCUP (SSSCUP). Therefore, we re-performed all of the aforementioned analysis on this subset of 88 patients. The univariable associations for OS and DFS related to HPV status and N stage seen in SSCUP remained apparent among sSSCUP patients. Furthermore, multivariable analysis for DFS showed that $\mathrm{cN} 3$ disease was associated with increased risk of DFS $(\mathrm{HR}=3.96, p=0.04)$ and both positive HPV status (HR=0.46, $p=0.05$ ) and treatment with CRT with or without LND (HR=0.39, $p=0.02$ ) were associated with decreased risk of DFS in sSSCUP (Table III). The similarity 

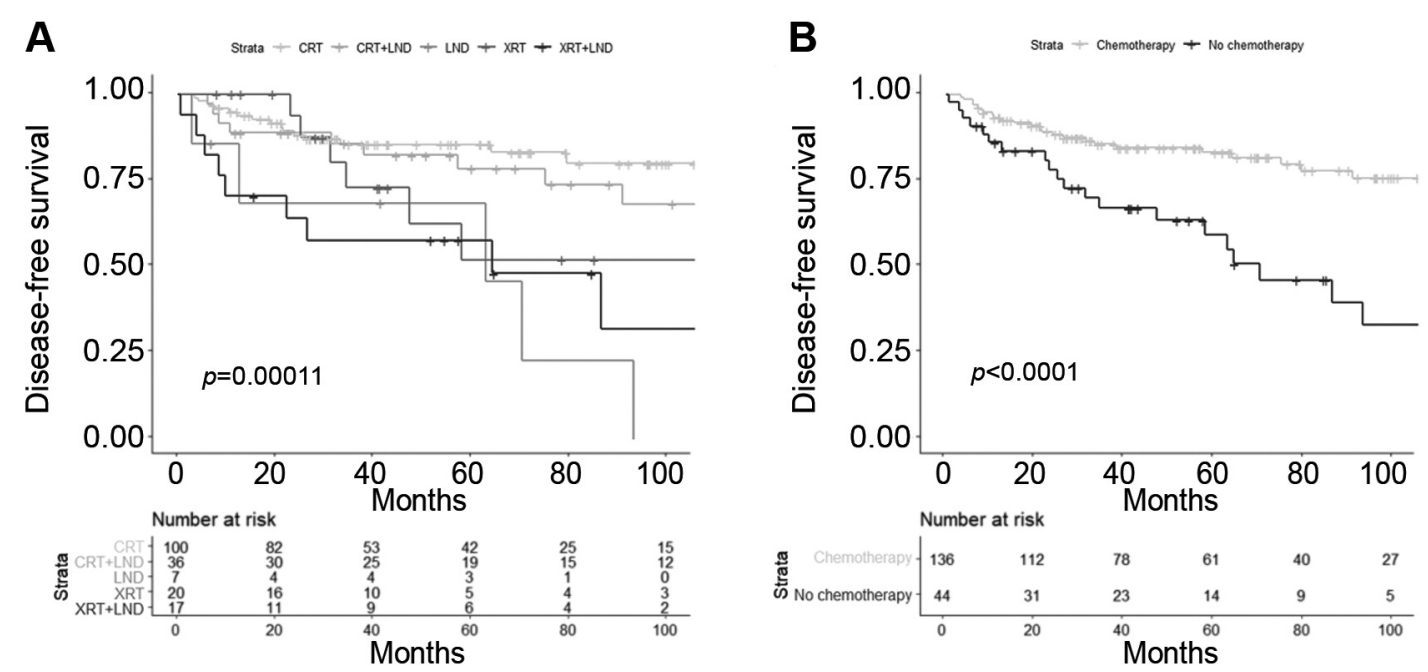

Figure 3. Disease free survival in all patients stratified by treatment regimen (A) and by whether the treatment regimen contained chemotherapy (B). Vertical dashes represent censored patients.
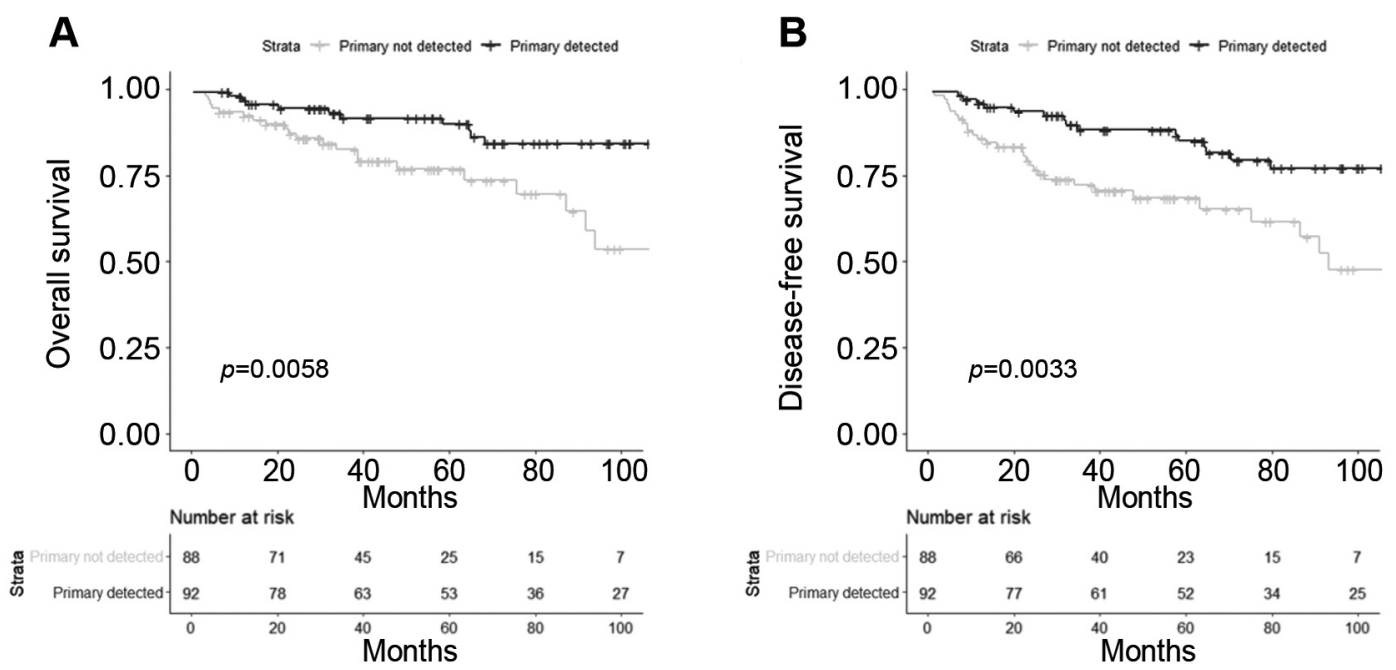

Figure 4. Overall (A) and disease free survival (B) in all patients stratified by whether or not the primary tumor site was detected. Vertical dashes represent censored patients.

of our results based on our definition of SCCUP provides evidence that our outcomes are robust.

\section{Discussion}

Finding the optimal approach to diagnosis and treatment of SCCUP is uniquely challenging because of the lack of prospective studies and definitive evidence in this disease. Often, clinicians rely on clinical judgement to determine the most appropriate treatment plan (13). The American Society of Clinical Oncology has published guidelines for the diagnosis and treatment of SCCUP that insightfully summarize the current data in the field (14). Our study, like previous studies, found a great majority of these tumors in the cryptic tissues of the oropharynx, with a heavy majority showing association with HPV (15).

In our study, the detection methodology was largely dependent on the individual caregivers. Generally, the most common approach, after initial in office examination, was a PET/CT scan followed by direct laryngoscopy including biopsies of candidate sites with or without surgery to remove the tonsils and lingual tonsils. This is in line with the most 
Table II. Multivariable cox regression analysis for disease free survival.

\begin{tabular}{lcccc}
\hline Factor & Hazard ratio & $2.5 \% \mathrm{CI}$ & $97.5 \% \mathrm{CI}$ & $p$-Value \\
\hline $\mathrm{cN} 1$ & Reference & - & - & - \\
$\mathrm{cN} 2$ & 1.22 & 0.52 & 2.88 & 0.64 \\
$\mathrm{cN} 3$ & 2.23 & 0.78 & 6.39 & 0.14 \\
HPV negative & Reference & - & - & - \\
HPV status not tested & 0.58 & 0.2 & 1.66 & 0.31 \\
HPV positive & 0.26 & 0.1 & 0.65 & 0.004 \\
Primary site detected & 0.72 & 0.37 & 1.42 & 0.25 \\
Treatment without CRT & Reference & - & - & - \\
CRT \pm LND & 0.38 & 0.19 & 0.74 & 0.004 \\
\hline
\end{tabular}

All model variables are included in the table with reference categories denoted. Data represented as hazards ratios along with $95 \%$ confidence intervals (CI) and $p$-Values. Hazard ratios above 1 represent greater hazard of progression or death.

current American Society of Clinical Oncology guidelines (14). Approximately half of the 133 patients who received a PET/CT demonstrated suspicious findings which directed biopsy sampling. With regards to biopsies, the most commonly biopsied sites were the oropharyngeal structures. The surface epithelium of the palatine and lingual tonsils is composed of deep invaginations into the lymphoid stroma, creating crypts that increase the surface area by nearly $700 \%$ (16). Within these crypts, the superficial cell layer is interrupted by migrating non-epithelial cells (such as lymphocytes) which cause breaks in the otherwise continuous barrier. This exposes the basement membrane, leaving it prone to deposition of viral particles (16). Therefore, these structures are increasingly susceptible to HPV infection. Unfortunately, the same cryptic organization also makes detection more challenging as small tumors may hide in the tonsillar folds, escaping detection.

Our primary detection rate of $51.1 \%$ is in line with previous studies. In a study of 110 patients, Ryan et al. identified the primary sites of $53 \%$ of tumors using a stepwise approach similar to the detection techniques used at our institution. Similar to our study, direct laryngoscopy identified a primary site in $31 \%$ of patients, while the rest were identified by palatine or lingual tonsillectomy (6). In our multivariable analysis, a lack of suspicious PET/CT findings was associated with lower odds of finding the primary site, perhaps indicating that these tumors are exceedingly small and difficult to locate. Previous studies have shown varying levels of utility for $\mathrm{PET} / \mathrm{CT}$ scans. Noij et al. found that quantitative analysis using $\mathrm{SUV}_{\max }$ resulted in a sensitivity and specificity of $81.3 \%$ and 93.3\% respectively, in a small sample of 31 patients (17). However, a larger analysis of 190 patients found a sensitivity and specificity of $73.1 \%$ and $68.9 \%$, respectively (18). In our study, sensitivity and specificity of PET/CT in ultimately detecting a cancer was $62.5 \%$ and $65.2 \%$, respectively. Nevertheless, further studies are needed in order to determine
Table III. Multivariable cox regression analysis for disease free survival among only patients where primary site was not detected prior to treatment.

\begin{tabular}{lcccc}
\hline Factor & Hazard ratio & $2.5 \% \mathrm{CI}$ & $97.5 \% \mathrm{CI}$ & $p$-Value \\
\hline $\mathrm{cN} 1$ & Reference & - & - & - \\
$\mathrm{cN} 2$ & 1.67 & 0.53 & 5.31 & 0.38 \\
$\mathrm{cN} 3$ & 3.96 & 1.1 & 14.3 & 0.04 \\
HPV negative or untested & Reference & - & - & - \\
HPV positive & 0.46 & 0.21 & 0.99 & 0.05 \\
Treatment without CRT & Reference & - & - & - \\
CRT \pm LND & 0.39 & 0.18 & 0.87 & 0.02 \\
\hline
\end{tabular}

All model variables are included in the table with reference categories denoted. Data represented as hazards ratios along with $95 \%$ confidence intervals (CI) and $p$-Values. Hazard ratios above 1 represent greater hazard of progression or death.

whether PET/CT is a useful tool in ultimately affecting treatment decisions and outcomes. It cannot be used as the definitive tool to determine a primary site given the data, and suggestive findings should be confirmed histologically.

Lingual tonsillectomy has arisen as a tool with great promise in detecting unknown primary sites. In our study, four of eight diagnostic lingual tonsillectomies were positive (50\%). All of these patients had their lingual tonsillectomy performed at the time of DL. In the majority of cases, the surgeon would preemptively discuss with the patient that a lingual tonsillectomy may be performed if no obvious tumor was discovered elsewhere. In other cases, a suspicious PET scan was the reason for lingual tonsillectomy. Ryan et al. reported eight primaries detected in 14 lingual tonsillectomies (57\%) and an increase in the total detection rate from $44 \%$ to $66 \%$, after introduction of lingual tonsillectomy to their institutional approach (6). In another study, Nagel et al. performed lingual tonsillectomy in 14 patients with negative biopsies, and detected eight primaries (57\%) (19). Together, these data suggest a role for TORS lingual tonsillectomy in patients where a primary is not detected after biopsies and palatine tonsillectomy, even when base of tongue biopsies are negative. However, it is important to keep in mind that lingual tonsillectomy is associated with significant morbidities including postoperative pain, decreased swallowing function, and high risk of readmission $(19,20)$.

OS and DFS were excellent in our cohort, with five-year OS and DFS of $85 \%$ and $77 \%$, respectively, driven by the high percentage of HPV associated disease. As with many previous studies, we found that HPV associated tumors carried a far better prognosis than HPV negative tumors (15, 21-23). DNA and mRNA evaluation in tumor samples has shown that a majority of HPV associated oropharyngeal tumors are HPV driven and that HPV DNA and p16 status are independent markers for prognosis (23). 
The treatment of SCCUP consists of varying permutations of chemotherapy, radiation therapy, and neck dissection. Many previous studies have suggested the equivalency of treatment across modalities (24-26). We found significant differences in OS and DFS across treatment methods in our cohort. Treatments with CRT offered the highest OS and DFS among treatments in our cohort. We noted that there seemed to be a clear separation in DFS between treatment regimens that included CRT with or without LND versus those that did not (Figure 3B). In fact, five year DFS among patients that received CRT was $83.2 \%$ compared to $59.3 \%$ in those that did not $(p<0.001$, log-rank test). However, it is important to keep these findings in context. At our institution, CRT is the standard of care for all patients who meet appropriate criteria. In patients who did not receive CRT, it must be presumed that there was a reason (perhaps a medical or other contraindication). Therefore, this group is likely to have done worse. Similarly, the most aggressive treatments were offered to the most robust patients. We were also unable to control for other factors such as performance status in our multivariable analysis, which is a well identified prognostic factor. Therefore, this finding warrants further investigation.

To analyze the effects of all of these variables on OS and DFS, we used multivariable Cox proportional hazards regression models. When controlling for $\mathrm{N}$ stage, HPV status, primary site detection, and treatment modality, we found that $\mathrm{cN} 3$ disease predicted worse OS and treatment with CRT predicted better OS. Similarly, we found that HPV positive status $(\mathrm{HR}=0.26)$ and treatment with CRT $(\mathrm{HR}=0.38)$ predicted better DFS (Table II). Together, these data confirm that HPV associated tumors have a substantially better prognosis as compared to HPV negative tumors, and that treatment of SCCUP with CRT offers excellent outcomes in patients who are eligible.

The definition of SCCUP is difficult to pinpoint; the most recent ASCO guidelines do not explicitly define head and neck SCCUP (14). Therefore, we performed our analysis using two different definitions of SCCUP: one being the lack of primary site after in-office fiberoptic examination, and the other being lack of primary site prior to treatment. Knowledge of a primary site prior to treatment allows for a more focused treatment, such as limiting the radiation field to the known tumor instead of a larger field. This may lead to decreased complications due to treatment. However, we found that our outcomes held true regardless of SCCUP definition, resulting in increased robustness and validity of our findings.

It is important to note the limitations associated with our study. Due to the inherent limitations of the retrospective study design, we were unable to control for many factors that factored into the choice of detection and treatment methods. For example, choice of treatment was likely influenced by a number of factors such as functional status of the patient or physician experience, which could have led to substantial selection bias in our cohort. Similarly, choice of biopsy location was likely dependent on surgeon experience and patient history not covered by our variables. Given the nonrandom nature of these variations, our results must be assessed in this context. Further studies, ideally in prospective form, should aim to further investigate the associations we have found in this study.

\section{Conclusion}

In our cohort of patients with SCCUP, we were able to detect a primary site in approximately half of patients. The majority of tumors were HPV associated and most detected tumors were located in the oropharynx. TORS lingual tonsillectomy is a promising tool and should be added to traditional detection methods in order to increase detection yield after directed biopsies and palatine tonsillectomy have failed to detect a primary site. When controlling for nodal stage, HPV positive tumor status, and treatment with CRT are significantly associated with better DFS although selection bias is likely present in our cohort.

\section{Conflicts of Interest}

The Authors declare no conflicts of interest.

\section{Authors' Contributions}

Study concept: MYL, NF, DA, SK, BP, BBB. Study design: MYL, DA, SK, BBB. Data acquisition: MYL. Data analysis and interpretation: MYL, DA, SK, BP, BBB. Manuscript preparation: MYL, NF, DA, SK, BP, BBB. Manuscript final approval: MYL, NF, DA, SK, BP, BBB.

\section{References}

1 Marur S and Forastiere AA: Head and neck cancer: changing epidemiology, diagnosis, and treatment. Mayo Clin Proc 83: 489-501, 2008. PMID: 18380996. DOI: 10.4065/83.4.489

2 Grau C, Johansen LV, Jakobsen J, Geertsen P, Andersen E and Jensen BB: Cervical lymph node metastases from unknown primary tumours. Results from a national survey by the Danish Society for Head and Neck Oncology. Radiother Oncol 55: 121-129, 2000. PMID: 10799723. DOI: 10.1016/s0167-8140(00)00172-9

3 Cuaron J, Rao S, Wolden S, Zelefsky M, Schupak K, Mychalczak B and Lee N: Patterns of failure in patients with head and neck carcinoma of unknown primary treated with radiation therapy. Head Neck 38: E426-E431, 2016. PMID: 25581274. DOI: 10.1002/hed.24013

4 Kwee TC and Kwee RM: Combined FDG-PET/CT for the detection of unknown primary tumors: systematic review and meta-analysis. Eur Radiol 19: 731-744, 2009. PMID: 18925401. DOI: $10.1007 / \mathrm{s} 00330-008-1194-4$

5 Rudmik L, Lau HY, Matthews TW, Bosch JD, Kloiber R, Molnar CP and Dort JC: Clinical utility of PET/CT in the evaluation of head and neck squamous cell carcinoma with an unknown primary: a prospective clinical trial. Head Neck 33: 935-940, 2011. PMID: 21674668. DOI: 10.1002/hed.21566 
6 Ryan JF, Motz KM, Rooper LM, Mydlarz WK, Quon H, Gourin CG, Tan M, Eisele DW and Fakhry C: The impact of a stepwise approach to primary tumor detection in squamous cell carcinoma of the neck with unknown primary. Laryngoscope 129: 16101616, 2019. PMID: 30565698. DOI: 10.1002/lary.27625

7 Mehta V, Johnson P, Tassler A, Kim S, Ferris RL, Nance M, Johnson JT and Duvvuri U: A new paradigm for the diagnosis and management of unknown primary tumors of the head and neck: a role for transoral robotic surgery. Laryngoscope 123: 146-151, 2013. PMID: 23154813. DOI: 10.1002/lary.23562

8 Chernock RD and Lewis JS: Approach to metastatic carcinoma of unknown primary in the head and neck: Squamous cell carcinoma and beyond. Head Neck Pathol 9: 6-15, 2015. PMID: 25804376. DOI: 10.1007/s12105-015-0616-2

9 Galloway TJ and Ridge JA: Management of squamous cancer metastatic to cervical nodes with an unknown primary site. J Clin Oncol 33: 3328-3337, 2015. PMID: 26351351. DOI: 10.1200/JCO.2015.61.0063

10 Compton AM, Moore-Medlin T, Herman-Ferdinandez L, Clark C, Caldito GC, Wang XI, Thomas J, Abreo FW and Nathan CAO: Human papillomavirus in metastatic lymph nodes from unknown primary head and neck squamous cell carcinoma. Otolaryngol Head Neck Surg 145: 51-57, 2011. PMID: 21493313. DOI: $10.1177 / 0194599811400385$

11 Tribius S, Hoffmann AS, Bastrop S, Görögh T, Haag J, Röcken C, Clauditz T, Grob T, Wilczak W, Tennstedt P, Borcherding A, Petersen $\mathrm{C}$ and Hoffmann M: HPV status in patients with head and neck of carcinoma of unknown primary site: HPV, tobacco smoking, and outcome. Oral Oncol 48: 1178-1184, 2012. PMID: 22739067. DOI: 10.1016/j.oraloncology.2012.05.022

12 Deschler DG, Richmon JD, Khariwala SS, Ferris RL and Wang MB: The "new" head and neck cancer patient-young, nonsmoker, nondrinker, and HPV positive: evaluation. Otolaryngol Head Neck Surg 151: 375-380, 2014. PMID: 24925311. DOI: $10.1177 / 0194599814538605$.

13 Adelstein D, Gillison ML, Pfister DG, Spencer S, Adkins D, Brizel DM, Burtness B, Busse PM, Caudell JJ, Cmelak AJ, Colevas AD, Eisele DW, Fenton M, Foote RL, Gilbert J, Haddad RI, Hicks WL, Hitchcock YJ, Jimeno A, Leizman D, Lydiatt WM, Maghami E, Mell LK, Mittal BB, Pinto HA, Ridge JA, Rocco J, Rodriguez CP, Shah JP, Weber RS, Witek M, Worden F, Yom SS, Zhen W, Burns JL and Darlow SD: NCCN Guidelines Insights: Head and Neck Cancers, Version 2, 2017. J Natl Compr Canc Netw 15: 761-770, 2017. PMID: 28596256. DOI: 10.6004/jncen.2017.0101

14 Maghami E, Ismaila N, Alvarez A, Chernock R, Duvvuri U, Geiger J, Gross N, Haughey B, Paul D, Rodriguez C, Sher D, Stambuk HE, Waldron J, Witek M and Caudell J: Diagnosis and management of squamous cell carcinoma of unknown primary in the head and neck: ASCO Guideline. J Clin Oncol: JCO2000275, 2020. PMID: 32324430. DOI: 10.1200/JCO.20.00275

15 Cheraghlou S, Torabi SJ, Husain ZA, Otremba MD, Osborn HA, Mehra S, Yarbrough WG, Burtness BA and Judson BL: HPV status in unknown primary head and neck cancer: Prognosis and treatment outcomes. Laryngoscope 129: 684-691, 2019. PMID: 30151832. DOI: $10.1002 /$ lary. 27475

16 Howard JD and Chung CH: Biology of human papillomavirusrelated oropharyngeal cancer. Semin Radiat Oncol 22: 187-193, 2012. PMID: 22687942. DOI: 10.1016/j.semradonc.2012.03.002
17 Noij DP, Martens RM, Zwezerijnen B, Koopman T, de Bree R, Hoekstra OS, de Graaf P and Castelijns JA: Diagnostic value of diffusion-weighted imaging and 18F-FDG-PET/CT for the detection of unknown primary head and neck cancer in patients presenting with cervical metastasis. Eur J Radiol 107: 20-25, 2018. PMID: 30292267. DOI: 10.1016/j.ejrad.2018.08.009

18 Sokoya M, Chowdhury F, Kadakia S and Ducic Y: Combination of panendoscopy and positron emission tomography/computed tomography increases detection of unknown primary head and neck carcinoma. Laryngoscope 128: 2573-2575, 2018. PMID: 30194842. DOI: 10.1002/lary.27268

19 Nagel TH, Hinni ML, Hayden RE and Lott DG: Transoral laser microsurgery for the unknown primary: role for lingual tonsillectomy. Head Neck 36: 942-946, 2014. PMID: 23616406. DOI: $10.1002 /$ hed.23372

20 Merna C, Lin HW and Bhattacharyya N: Clinical characteristics, complications, and reasons for readmission following lingual tonsillectomy. Otolaryngol Head Neck Surg 160: 619-621, 2019. PMID: 30776976. DOI: 10.1177/0194599819827820

21 Axelsson L, Nyman J, Haugen-Cange H, Bove M, Johansson L, De Lara S, Kovács A and Hammerlid E: Prognostic factors for head and neck cancer of unknown primary including the impact of human papilloma virus infection. J Otolaryngol Head Neck Surg 46: 45, 2017. PMID: 28601094. DOI: 10.1186/s40463-017-0223-1

22 Hong AM, Dobbins TA, Lee CS, Jones D, Harnett GB, Armstrong BK, Clark JR, Milross CG, Kim J, O'Brien CJ and Rose BR: Human papillomavirus predicts outcome in oropharyngeal cancer in patients treated primarily with surgery or radiation therapy. Br J Cancer 103: 1510-1517, 2010. PMID: 20959828. DOI: $10.1038 /$ sj.bjc.6605944

23 Sivars L, Landin D, Grün N, Vlastos A, Marklund L, Nordemar S, Ramqvist T, Munck-Wikland E, Näsman A and Dalianis T: Validation of human papillomavirus as a favourable prognostic marker and analysis of CD8+ tumour-infiltrating lymphocytes and other biomarkers in cancer of unknown primary in the head and neck region. Anticancer Res 37: 665-673, 2017. PMID: 28179315. DOI: 10.21873/anticanres.11362

24 Demiroz C, Vainshtein JM, Koukourakis GV, Gutfeld O, Prince ME, Bradford CR, Wolf GT, McLean S, Worden FP, Chepeha DB, Schipper MJ and McHugh JB: Head and neck squamous cell carcinoma of unknown primary: neck dissection and radiotherapy or definitive radiotherapy. Head Neck 36: 15891595, 2014. PMID: 23996575. DOI: 10.1002/hed.23479

25 Shoushtari A, Saylor D, Kerr K-L, Sheng K, Thomas C, Jameson M, Reibel J, Shonka D, Levine P and Read P: Outcomes of patients with head-and-neck cancer of unknown primary origin treated with intensity-modulated radiotherapy. Int J Radiat Oncol Biol Phys 81: e83-91, 2011. PMID: 21377283. DOI: 10.1016/j.ijrobp.2011.01.014

26 Balaker AE, Abemayor E, Elashoff D and St John MA: Cancer of unknown primary: does treatment modality make a difference? Laryngoscope 122: 1279-1282, 2012. PMID: 22538837. DOI: $10.1002 /$ lary.22424

Received June 8, 2020

Revised June 29, 2020

Accepted July 1, 2020 